\title{
Subdiffraction scattered light imaging of gold nanoparticles using structured illumination
}

\author{
Bo-Jui Chang, ${ }^{1}$ Shiuan Huei Lin, ${ }^{2}$ Li-Jun Chou, ${ }^{1}$ and Su-Yu Chiang ${ }^{1, *}$ \\ ${ }^{1}$ National Synchrotron Radiation Research Center, 101 Hsin-Ann Road, Hsinchu Science Park, Hsinchu 30076, Taiwan \\ ${ }^{2}$ Department of Electrophysics, National Chiao Tung University, Hsinchu 300, Taiwan \\ *Corresponding author: schiang@nsrrc.org.tw \\ Received July 22, 2011; revised September 28, 2011; accepted October 20, 2011; \\ posted November 2, 2011 (Doc. ID 151451); published December 14, 2011

\begin{abstract}
A reflective light-scattering (RLS) microscope with structured illumination (SI) provides subdiffraction resolution and improves the image quality of gold nanoparticles in biological systems. The three-dimensional (3D)-structured pattern is rapidly and precisely controlled with a spatial light modulator and scrambled at the conjugate image plane to increase spatial incoherence. The reconstructed SI-RLS image of $100 \mathrm{~nm}$ gold nanoparticles reveals lateral and axial resolutions of approximately 117 and $428 \mathrm{~nm}$. We present a high-resolution image of gold nanoparticles inside a HeLa cell, with improved contrast. () 2011 Optical Society of America

OCIS codes: $180.0180,180.6900$.
\end{abstract}

The increasing importance of gold nanoparticles in biological and biomedical applications makes scattered light imaging an attractive modality to investigate their behavior and interactions in cells. Apart from having excellent biocompatibility and stability, gold nanoparticles feature both a strong ability to scatter light and resistance to photobleaching [1]. The use of gold nanoparticles of size $40-200 \mathrm{~nm}$ as optical probes for dark-field imaging has enabled long-term tracking of the movements of molecular motors [2,3]. Combined with scattering spectra, the spectral imaging and tracking ability of a single $80 \mathrm{~nm}$ gold nanoparticle in vivo have been exploited [4]. However, the achievable spatial resolution in these studies remains limited by the diffraction nature of light.

Conventional light-scattering microscopic techniques use various schemes, such as differential interference contrast and dark-field illumination, to enhance the image contrast but barely improve the resolution. For a microscope, several super-resolution fluorescence techniques, such as stimulated emission depletion (STED) microscopy, stochastic optical reconstruction microscopy (STORM), and three-dimensional (3D)-structured illumination microscopy (3D-SIM), have been developed based on various excitation strategies [5-7]. Among them, 3D-SIM is an attractive method to improve both lateral and axial resolution with true optical sectioning through merely altering the illumination pattern [7]. The structured illumination of samples enables undetectable high-frequency information to be encoded into a detectable region and then computationally extracted to extend the optical-transfer function (OTF) of a wide-field microscope, so improving the resolution.

In preceding 3D-SIM fluorescence imaging, a diffraction grating serves to generate illumination patterns, but the mechanical motion of a grating is time-consuming [?]. A spatial light modulator (SLM) can overcome this shortcoming; moreover, the design of SLM patterns enables one to adjust the contrast of structured illumination to improve the image contrast. The use of a SLM has been demonstrated for 2D-SIM fluorescence imaging [몰] but not for 3D-SIM imaging.

In this Letter, we adopted 3D-SIM to the scattered light imaging of gold nanoparticles to achieve nearly doubled lateral and axial resolutions, concurrently improving the image contrast. We implemented a SLM to generate the 3D-structured patterns rapidly and precisely. To avoid speckle scattering from a sample and complicated coherent image retrieval, the spatial coherence of the illumination pattern was scrambled at the conjugate image plane with a holographic diffuser. The reflective lightscattering (RLS) microscope with structured illumination is abbreviated as a SI-RLS microscope. We present a SI-RLS image of $100 \mathrm{~nm}$ gold nanoparticles inside a HeLa cell at subdiffraction resolution with improved contrast.

Figure 1 shows the setup of the SI-RLS microscope. The mainframe is an upright microscope with a He-Ne laser operating at $543 \mathrm{~nm}$. The incident laser beam is expanded and diffracted with a phase-only SLM (X1046804 , Hamamatsu) into laser beams of orders 0 and \pm 1 that are then focused with five lenses in a set onto the back focal plane (pupil) of an objective (Plan Apochromat VC $60 \times / 1.2$ WI, Nikon). The laser beams of orders \pm 1 are focused near the opposite edges of the pupil plane and that of zero-order is at the center; the beams intersect each other in the focal plane of the objective to form a 3D-structured pattern. To generate the illumination patterns at four orientations of nearly the same contrast, a

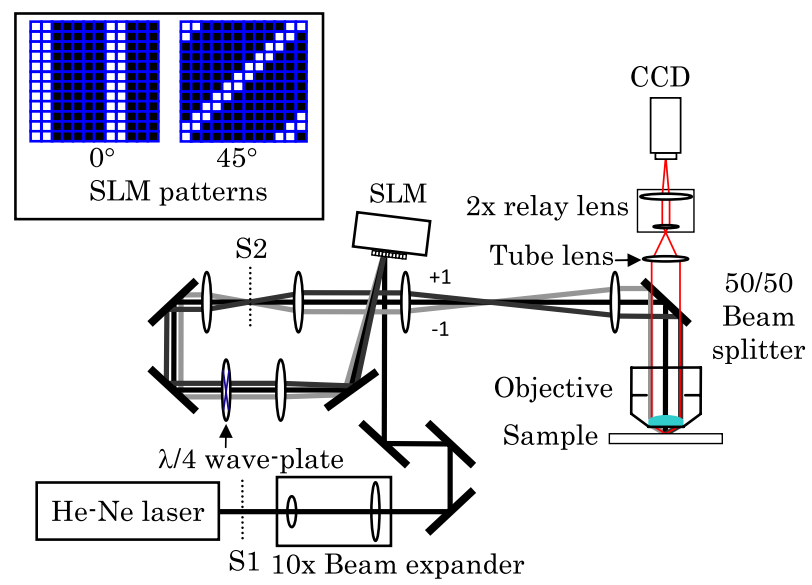

Fig. 1. (Color online) Schematic of the SI-RLS microscope. (Inset) Two SLM patterns designed to generate $0^{\circ}$ and $45^{\circ}$ illumination patterns. 
$\lambda / 4$ wave plate is placed after the SLM to produce circularly polarized light. The use of circularly polarized light rather than $s$-polarized light will yield a weak contrast but simplify the microscopic system. The scattered light is collected and then detected with an electron-multiplying CCD camera (DU885, Andor). The axial position of the sample is controlled with a stage driven with a piezoelectric transducer (P-562.3CD, PI).

The design of the SLM patterns is classified into two sets, $\left(0^{\circ}, 90^{\circ}\right)$ and $\left(45^{\circ}, 135^{\circ}\right)$, to generate $3 \mathrm{D}$-structured patterns; the lateral periods are 274 and $277 \mathrm{~nm}$, respectively. The inset of Fig. 1 shows two SLM patterns designed in periods of length 7 and $5 \sqrt{ } 2$ pixels (diagonally) to generate $0^{\circ}$ and $45^{\circ}$ illumination patterns. On shifting one pixel for each SLM pattern, the corresponding illumination patterns shift laterally $4 \pi / 7$ and $4 \pi / 5$ in phase, respectively. By setting $0.875 \pi$ phase retardation in the white pixels, we obtain nearly the same ratios, 0.83 , $0.89,0.92$, and 0.9 , for the light of zero- and first-orders to generate $0^{\circ}, 45^{\circ}, 90^{\circ}$, and $135^{\circ}$ illumination patterns.

To reconstruct a 3D high-resolution SI-RLS image, intermediate SI images in stacks are acquired at five phases $-\phi_{m}=4(m-1) / 7+\phi_{0}$ or $4(m-1) / 5+\phi_{0}$ with $m=1-5$ and $\phi_{0}$ the initial phase of the pattern and four pattern orientations at a selected focus step. The image reconstruction follows the pioneer work of Gustafsson et al. [7]. Briefly, the 3D Fourier transform of intermediate SI stacks taken at five phases at each pattern orientation is used to retrieve five information bands that correspond to the five lateral illumination-intensity frequencies; two of them are shared by pairs. These five bands consist of the conventional OTF and the high-frequency information components in the lateral and axial directions. After twenty bands at four pattern orientations are retrieved, we average the four OTF before combining them with the sixteen new bands and then use a Wiener deconvolution to form a total 3D-SI spectrum $D_{\mathrm{SI}}(k)$. The inverse Fourier transform of $D_{\mathrm{SI}}(k)$ yields a high-resolution SIRLS image.

The coherence of the laser beams causes two difficulties in retrieving a SI-RLS image: the reflections from various interfaces produce a strong and inhomogeneous background to preclude a satisfactory differentiation between scattered light signals and background and the coherent image retrieval involves complicated algorithms. Hence, we scramble the spatial coherence of the illumination source with a rotating holographic diffuser (U47988, Edmund Optics). To evaluate the effect of spatial incoherence on the image, two locations are selected, $\mathrm{S} 1$ and S2, indicated in Fig. 1; S1 is near the output of the laser beam whereas $\mathrm{S} 2$ is at the conjugate image plane of the 3D-structured pattern.

With merely the central zero-order light for illumination, Fig. 2 compares the projected wide-field images of $100 \mathrm{~nm}$ gold nanoparticles immersed in water on a coverslip with scrambling at (a) S1 and (b) S2. The image in Fig. 2(a) evidently suffers from an axially periodic background that results mainly from the multiple reflections on various interfaces; accordingly, the illumination retains some degree of coherence on scrambling at S1. In contrast, the background in Fig. 2(b) is negligible, comparable to that from illumination with a halogen lamp, as shown in Fig. 3(a), indicating that an effective
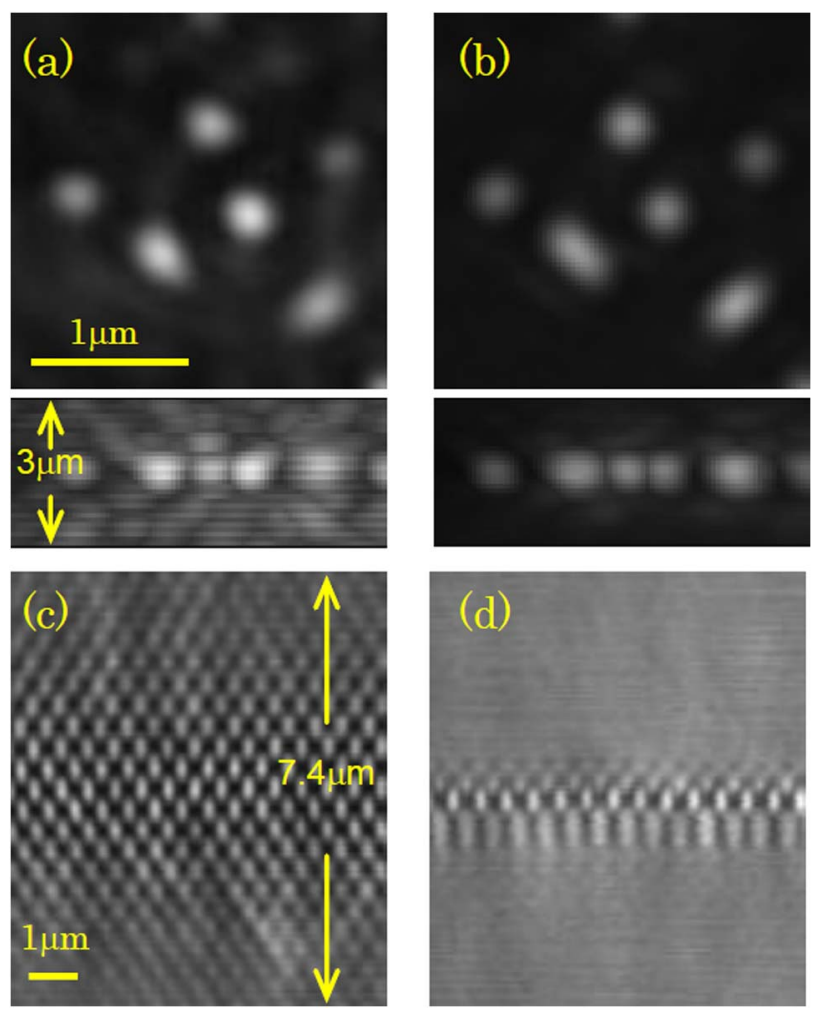

Fig. 2. (Color online) Projections of wide-field images of $100 \mathrm{~nm}$ gold nanoparticles on scrambling at locations (a) S1 and (b) S2. Each stack contains 31 images taken at $z$-step of $100 \mathrm{~nm}$; the upper and lower parts represent the lateral $(x y)$ and axial $(x z)$ projections, respectively. The axial extents of the 3D-structured patterns are shown with scrambling at (c) S1 and (d) S2.

scrambling at S2 leads to the production of a quasiincoherent image.

To rationalize the imaging on scrambling at S2, we describe the amplitude of scattered signals on the CCD plane as
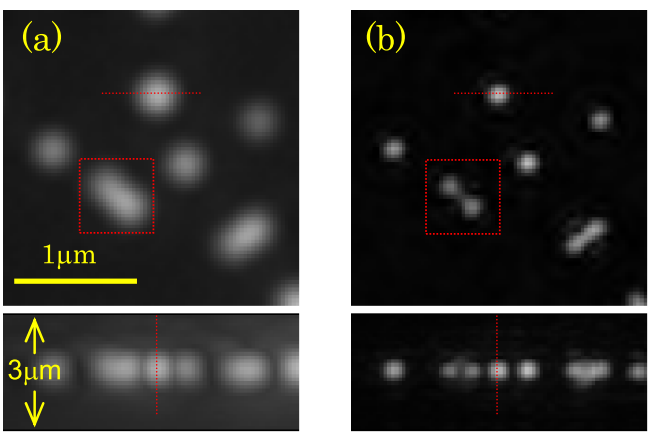

(c)
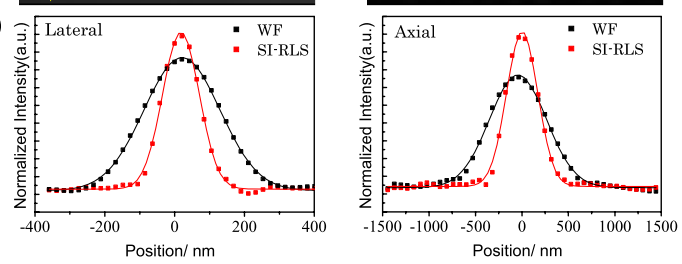

Fig. 3. (Color online) Projections of $100 \mathrm{~nm}$ gold nanoparticles from (a) wide-field and (b) SI-RLS images in stacks. (c) Lateral and axial profiles of a single gold nanoparticle, with a Gaussian fit. Each stack contains 31 images taken at $z$-step of $100 \mathrm{~nm}$. 


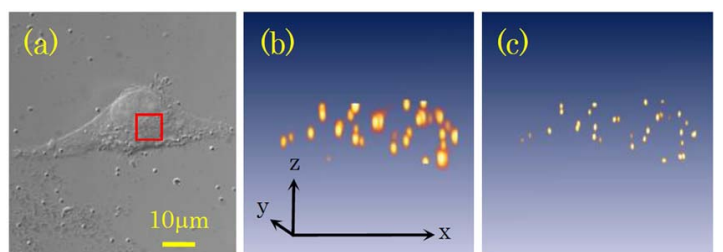

Fig. 4. (Color online) (a) DIC image of an internalized cell. 3D-view of (b) wide-field and (c) SI-RLS images of $100 \mathrm{~nm}$ gold nanoparticles within the red box. The stack in (b) and (c) contains 51 images taken at $z$-step of $100 \mathrm{~nm}$ with an image size of $7.7 \times 7.7 \mu \mathrm{m}^{2}$.

$$
\begin{aligned}
U_{i}(x, y, t) & =U_{g}(x, y, t) \otimes h(x, y) \\
& =\left[\sqrt{S(x, y)} \cdot E(x, y) \cdot e^{i \phi(t)}\right] \otimes h(x, y),
\end{aligned}
$$

in which appear the distribution of scattered signals on the sample plane $U_{g}(x, y, t)$, coherent point spread function $h(x, y)$, reflective intensity in the image of gold nanoparticles $S(x, y)$, amplitude of the illuminating light $E(x, y)$, and phase distribution $\phi(t)$ induced by a rotating diffuser. As the diffuser rotates rapidly during the exposure for imaging, the average of $\phi(t)$ is zero. The detected signal is thus the temporal average of the intensity of the scattered signal, described as [10]

$$
\begin{aligned}
I_{i}(x, y) & =\left\langle\left|U_{i}(x, y, t)\right|^{2}\right\rangle \\
& =\left[S(x, y)|E(x, y)|^{2}\right] \bigotimes|h(x, y)|^{2} .
\end{aligned}
$$

This equation corresponds to an incoherent image, consistent with the experimental observation.

Figures 2(c) and 2(d) compare the 3D-structured pattern on scrambling at S1 and $\mathrm{S} 2$, respectively; the images in a stack are taken at $z$-step of $100 \mathrm{~nm}$ by placing a silicon wafer on the sample stage. In contrast to Fig. 2(c) in which the pattern is an incoherent superposition of coherent point triplets around the sample plane, the pattern in Fig. 2(d) is the imaging of the scrambled 3D-structured pattern at the conjugate image plane as an effective scrambling at S2 leads to a nearly complete incoherent illumination. The observed axial extent of the modulation in Fig. 2(d) is comparable to the axial OTF of the microscope, but the contrast of about 0.5 is weak relative to 0.75 in Fig. 2(c).

The scrambling at $\mathrm{S} 2$ offers the feasibility of 3D-SIM imaging using incoherent image processes. Figure 3 shows the projections of (a) wide-field and (b) SI-RLS images of $100 \mathrm{~nm}$ gold nanoparticles. The SI-RLS image reveals an improved image contrast and superior resolution in both lateral and axial directions. Figure 3(c) compares the lateral and axial profiles of individual gold nanoparticles; the solid lines represent Gaussian-fits. The resolution improvement is nearly isotropic, with a FWHM difference less than $10 \%$ at four orientations. We obtain the average lateral and axial FWHM of $262 \pm 6$ and $867 \pm$ $19 \mathrm{~nm}$ from the wide-field image and of $117 \pm 10$ and $428 \pm 18 \mathrm{~nm}$ from the SI-RLS image, respectively. The resolution is thus improved by factors of $\sim 2.2$ laterally and $\sim 2.0$ axially. This improvement is comparable to that in 3D-SIM fluorescence imaging; a factor slightly more than two may be attributed to the Wiener deconvolution that boosts spatial frequencies in the high-frequency region, as explained by Gustafsson et al. [7]. However, we found that adjacent gold nanoparticles are less well resolved than fluorescence beads; residual signals in the red box remain discernible between the two gold nanoparticles. The locally enhanced electromagnetic field between gold nanoparticles might be responsible for this unsatisfactory separation [11].

To demonstrate the biological applications, we acquired a 3D image of $100 \mathrm{~nm}$ gold nanoparticles internalized with a HeLa cell. Cells were incubated in a Dulbecco's modified Eagle's medium solution without serum but with gold nanoparticles for cellular uptake. After incubation for $4 h$, we washed the cells with phosphate buffered saline (PBS) solution and then fixed them with paraformaldehyde (4\%) before immersion in water for imaging. Figure 4(a) shows the differential inference contrast (DIC) image of the cell. Within the red box, a $3 \mathrm{D}$ view of the wide-field image illuminated with a halogen lamp and the SI-RLS image are shown in Figs. 4(b) and $\underline{4(\mathrm{c})}$ for comparison. The SI-RLS image achieves a decreased background and improves the resolution to enable the differentiation of adjacent nanoparticles.

In summary, we demonstrate the adoption of a SLM-based 3D-SIM to scattered light imaging of gold nanoparticles in biological specimens using a scrambled 3D-structured pattern. The scrambling at the conjugate image plane appreciably improves the intermediate SI images and enables use of an incoherent imaging model. We expect that the improved performance of our SI-RLS system will be helpful to researchers employing nanoparticle-based imaging in biological systems. Considering the small duration of exposure-typically $<5 \mathrm{~ms}$ for one intermediate SI image and no photobleaching in imaging, a SLM-based SI-RLS system also makes feasible 3D SI-RLS imaging in real time for the observation of nanoparticles motions in living cells.

The National Synchroton Radiation Research Center (NSRRC) and the National Science Council of Taiwan (NSCT) (NSC99-2113-005-MY2) provided financial support.

\section{References}

1. R. A. Sperling, P. R. Gil, F. Zhang, M. Zanekka, and W. Parak, Chem. Soc. Rev. 37, 1896 (2008).

2. X. Nan, P. A. Sims, and X. S. Xie, Chem. Phys. Chem. 9, 707 (2008).

3. H. Ueno, S. Nishikawa, R. Iino, K. V. Tabata, S. Sakakihara, T. Yanagida, and H. Noji, Biophys. J. 98, 2014 (2010).

4. G. Louit, T. Asahi, G. Tanaka, T. Uwada, and H. Masuhara, J. Phys. Chem. C 113, 11766 (2009).

5. B. Hein, K. I. Willig, and S. W. Hell, Proc. Natl. Acad. Sci. USA 105, 14271 (2008).

6. B. Huang, W. Wang, M. Bates, and X. Zhuang, Science 319, 810 (2008).

7. M. G. L. Gustafsson, L. Shao, P. M. Cariton, C. J. R. Wang, I. N. Golubovskaya, W. Z. Cande, D. A. Agard, and J. W. Sedat, Biophys. J. 94, 4957 (2008).

8. P. Kner, B. B. Chhun, E. R. Griffis, L. Winoto, and M. G. L. Gustafsson, Nat. Methods 6, 339 (2009).

9. M. R. Beversluis, G. W. Bryant, and S. J. Stranick, J. Opt. Soc. Am. A 25, 1371 (2008).

10. J. W. Goodman, Introduction to Fourier Optics (McGrawHill, New York, 1968), Chap. 6.

11. H.-Y. Lin, C.-H. Huang, C.-H. Chang, Y.-C. Lan, and H.-C. Chui, Opt. Express 18, 165 (2009). 